\title{
- Clinical Research • \\ Clinical features and survival analysis of different subtypes of patients with breast cancer brain metastases
}

\author{
Bing Bai1 ${ }^{1,2}$, Zhong-Yu Yuan ${ }^{1,2}$, Dong-Geng Liu' ${ }^{1,2}$, Xiao-Yu Teng ${ }^{1,2}$, Shu-Sen Wang ${ }^{1,2}$ \\ ${ }^{1}$ State Key Laboratory of Oncology in South China, Guangzhou, Guangdong 510060, P. R. China; ${ }^{2}$ Department of Medical Oncology, Sun Yat-sen \\ University Cancer Center, Guangzhou, Guangdong 510060, P. R. China
}

【Abstract】 Background and Objective: The brain is one of the most common metastatic sites of breast cancer. Brain metastases develop in $10 \%-15 \%$ of patients with breast cancer and are associated with poor prognosis. The purpose of this retrospective study was to analyze the clinical characteristics and survival of patients with brain metastases due to breast cancer of different subtypes and to identify the prognostic factors that affect clinical outcome. Methods: A total of 89 patients with breast cancer brain metastases diagnosed between October 1997 and July 2008 at Sun Yat-sen University Cancer Center were included in this study. Among the 89 patients, the number of luminal A, luminal B, human epidermal growth factor receptor 2 (HER-2), and triple-negative (TN) subtypes were $30,20,16$, and 14, respectively; 9 patients had an unknown subtype. The clinical characteristics, pathologic features, and prognostic factors were analyzed both at the initial diagnosis and at the diagnosis of brain metastases. Endocrine therapy for patients with luminal subtypes was further studied. Results: The median age of patients was 46 years (range 28-74 years). The median survival time was 8.0 months (range, 0-80 months), the 1-year survival rate was 32\% and the 5year survival rate was $4 \%$. The time to brain metastasis differed according to clinical stage at the initial diagnosis, and the time for patients with the luminal A subtype was the longest $(P<0.001)$. Multivariate analysis demonstrated that performance status score $>$ 1 , multiple brain metastases and without whole brain radiotherapy (WBRT) in combination with chemotherapy were associated with poor prognosis. Compared with the luminal A subtype, features of the HER-2 and TN subtypes included early metastases, rapid progression after first-line treatment (8.0 months vs. 11.0 months), and poor overall survival (25.0 months vs. 63.0 months). The luminal A subtype showed a tendency for good prognosis and slow growth. Tamoxifen could improve the survival of luminal A/B subtypes (median survival 24.0 months vs. 7.0 months, respectively, $P=0.002$ ). Conclusions: The prognosis of brain metastases from breast cancer was poor, especially in patients with HER-2 and TN subtypes. Generally, WBRT in combination with chemotherapy was the standard treatment modality. Patients with the luminal subtypes could benefit from tamoxifen.

Key words: Breast cancer, brain metastases, survival, prognosis, HER-2, triple negative

Breast cancer is a common cancer in women with high mortality. With advances in diagnostic techniques and the application of new treatments, such as targeted therapy for HER-2 amplification, the systemic disease control rate has been improved and the overall survival rate has been extended, while brain metastases are increasingly more common. Symptomatic brain metastases account for $10 \%$ to $16 \%$ of all patients with breast cancer, and the patients with brain metastases confirmed

\footnotetext{
Correspondence to: Shu-Sen Wang; Tel: +86-20-61639754; Tel: +86-20-61639754; Email: wangshs@mail.sysu.edu.cn

This paper was translated from Chinese into English by CJC Medical Translation and edited by Hope J. Lafferty on 2010-02-04.

The Chinese version of this paper is avaiable at http://www.cjcsysu.cn/cn/article .asp? id=16721.
}

Received: 2009-11-05; Accepted: 2009-12-21 by autopsy account for about $30 \%^{[1-4]}$. Brain metastases from breast cancer result in high mortality and have become a major factor leading to the decline in survival. There are different subtypes of breast cancer, and research suggests that simultaneous negative expressions of estrogen receptor (ER), progesterone receptor (PR), and human epidermal growth factor receptor 2 (HER-2), and HER-2 overexpression are risk factors for brain metastases occurring in patients with breast cancer ${ }^{[5-11]}$. Whole-brain radiotherapy (WBRT), surgery, stereotactic radiosurgery (SRS), chemotherapy, and targeted therapy are the common treatments for brain metastases from breast cancer. The purpose of this study was to analyze the clinicopathologic characteristics of different subtypes of patients with breast cancer with brain metastases and prognostic factors, to provide a reference to improve the prevention and treatment of brain metastases from breast cancer. 


\section{Patients and Methods}

\section{Clinical data}

The clinical data of 89 patients with brain metastases from breast cancer treated at Sun Yat-sen University Cancer Center between October 1997 and July 2008 were collected, including the performance status, pathologic diagnosis, tumor stage, treatment, the time and location of metastases, as well as the $E R, P R$, and HER-2 status. All patients were women and the median age at first visit was 44 years (26-67 years). Of these patients, $73.0 \%$ (65) had not reached menopause. Regarding pathologic type, $87.6 \%$ (78 patients) had invasive ductal carcinoma, $2.3 \%$ (2 patients) had invasive lobular carcinoma, and $10.1 \%$ (9 patients) had an unknown pathologic type. The histologic grade for $89.9 \%$ (80 patients) was grade II or III. In 12 patients with newly diagnosed stage-IV disease, 9 patients had multiple metastases, including to bone or visceral organs, 2 had brain metastases, and 1 had distant lymph node metastases alone.

All patients had pathologic diagnoses of primary tumors and were staged by the 7th edition of the American Joint Committee on Cancer (AJCC) breast cancer tumor node metastasis (TNM) staging system. HER-2 gene overexpression was defined as +++ by immunohistochemistry, or + by fluorescence in situ hybridization (FISH), or + by chromogenic in situ hybridization $(\mathrm{CISH})$. According to immunohistochemical indicators, patients with breast cancer were divided into four subtypes: luminal type $A$ was defined as ER-positive or PR-positive and HER-2-negative; luminal type $B$ was defined as ER-positive or PR-positive and HER-2-positive; the HER-2 overexpression type was defined as ER- and PR-negative and HER-2-positive; and triple-negative (TN) breast cancer was defined as ER-, PR-, and HER-2-negative. Because the immunohistochemical results of 9 patients were unknown, 80 patients with immunohistochemical results were classified based on the above definition, including $37.5 \%$ (30 patients) with luminal type A , 25.0\% (20 patients) with luminal type B, $20.0 \%$ (16 patients) with HER-2 overexpression, and $17.5 \%$ (14 patients) with TN. Brain metastasis was diagnosed by clinical symptoms, signs, and cranial magnetic resonance imaging (MRI) or computer tomography (CT).

\section{Treatment}

In the 89 patients, 12 patients with newly diagnosed stage-IV disease received chemotherapy containing anthracycline and taxane as first-line treatment, of whom 5 patients received palliative breast resection and 6 patients received radiotherapy. In 77 patients with newly diagnosed stage-I to -III disease, $80.5 \%$ (62 patients) received modified radical mastectomy and $19.5 \%$ (15 patients) received tumor resection. Of these patients, $94.8 \%$ (73 patients) received either adjuvant or neoadjuvant chemotherapy, of whom $56.2 \% \quad$ (41 patients) received anthracycline-based chemotherapy, 26.0\% (19 patients) received taxane and anthracycline combined chemotherapy, and $17.8 \%$ (13 patients) received fluorouracil, cyclophosphamide, methotrexate, capecitabine, vinorelbine, platinum, or other drug treatments. For other therapies, $51.9 \% \quad$ (40 patients) received adjuvant radiotherapy, including to the chest wall and the supraclavicular area, $42.9 \%$ (33 patients) received adjuvant endocrine therapy, and 1 patient with HER-2-positive expression received adjuvant trastuzumab therapy.

After being diagnosed with brain metastases, in the 89 patients, $23.6 \%$ (21 patients) had symptoms of central nervous system compression and received hormone, mannitol, or other symptomatic supportive treatments to reduce brain edema and alleviate the symptoms of intracranial hypertension. A total of $73.0 \%$ (65 patients) received WBRT of 30-40 Gy in 10 fractions by high energy $\mathrm{X}$-ray. One patient received $\gamma$-knife treatment. Almost half of patients $(47.2 \% \quad$ (42 patients $))$ received systemic chemotherapy, including taxane (20 patients, $22.5 \%)$, capecitabine (20 patients, $22.5 \%$ ), anthracycline (13 patients, $14.6 \%$ ), vinorelbine (16 patients, $17.9 \%$ ), gemcitabine (12 patients, $13.5 \%$ ), and platinum-group compounds (7 patients, $7.9 \%$ ), for between 2 to 8 cycles. A total of $21.3 \%$ (19 patients) received endocrine therapy, of whom 15 patients received tamoxifen and 4 patients received aromatase inhibitors (anastrozole, letrozole, or exemestane). As for other treatments, $14.6 \%$ (13 patients) received targeted therapy, of whom 6 patients received trastuzumab, 2 patients received lapatinib, 2 patients received bevacizumab, and 3 patients received erlotinib or gefitinib. In this study, no patients with brain metastases received surgery.

\section{Observation and follow-up}

Follow-up began when the patients were diagnosed with breast cancer. All patients were followed until July 31, 2009. Survival time and survival rate were calculated since the diagnosis of brain metastases.

\section{Statistical analysis}

SPSS version 17.0 was used for statistical processing. Survival analysis was performed by the Kaplan-Meier method and tested by log-rank test. Multivariate analysis was performed by the Cox proportional hazards model. Continuous variables were tested by $t$ test or an analysis of variance, and nonparametric tests were conducted by $\chi^{2}$ test. $P<0.05$ was considered statistically significant.

\section{Results}

\section{The clinical characteristics of patients with brain metastases}

All 89 patients with brain metastases were women, accounting for $11.4 \%$ of 780 patients with recurrent or metastatic breast cancer in the same period. The median disease-free interval from the first consultation to the first recurrence or metastasis was 20.0 months (0-127.0 months). The most common site of first recurrence or metastasis was bone (43 patients, $48.3 \%$ ), followed by lung or pleura (41 patients, $46.1 \%$ ), and brain (25 patients, $28.1 \%$ ). The median time from the first diagnosis to the diagnosed brain metastases was 30.0 months (0-128.0 months). In addition to 2 patients with newly diagnosed brain metastases, brain metastases in 9 patients 
$(10.3 \%)$ with newly diagnosed stage-I disease developed at a median time of 66.5 months (95\% Cl: 33.01-131.21 months), for 36 patients $(41.4 \%)$ with stage-Il disease the median time was 34.0 months ( $95 \% \mathrm{Cl}: 31.38-51.23$ months), for 32 patients $(36.8 \%)$ with stage-III disease the median time was 34.0 months (95\% Cl: 29.40-55.42 months), and for 10 patients (11.5\%) with stage-IV disease the median time was 10.0 months $(95 \% \mathrm{Cl}$ : 6.49-16.11 months $) \quad(P<0.001)$. The median age to be diagnosed with brain metastases was 46 years (28-74 years), with a median performance status score of 1 point (0 to 3 points). Regarding first, second, third, and fourth recurrence, 25.8\% (23 patients), 42.7\% (38 patients), 15.7\% (14 patients), and $13.5 \%$ (12 patients) had brain metastases, respectively. The most common site of accompanied metastases after the diagnosis of brain metastases was lung or pleura (57 patients, $64.1 \%$ ), followed by bone (55 patients, $61.8 \%$ ). Other metastatic sites included local or distant lymph nodes (43 patients, $48.3 \%$ ), liver (34 patients, 38.2\%), local recurrence (28 patients, $31.5 \%$ ), skin or soft tissue (18 patients, $20.2 \%$ ), contralateral breast (10 patients, $11.2 \%$ ), and other sites (4 patients, $4.5 \%$ ). Age at first diagnosis, time from first diagnosis to brain metastases, and time to progression after first-line treatment in different patient subtypes showed significant differences (Table 1).

\section{Survival of patients with brain metastases}

All patients were followed until July 31,2009 , with a median follow-up of 41.0 months (6.0-141.0 months). At the follow-up cutoff, $83.2 \%$ (74 patients) had died, $14.6 \%$ (13 patients) survived, and 2 patients were lost to follow-up. The median survival time of patients with brain metastases was 8.0 months (0-80.0 months, $95 \% \mathrm{Cl}$ : $6.57-9.42$ months), the 1-year survival rate was $32 \%$, the 2 -year survival rate was $17 \%$, and the 5 -year survival rate was $4 \%$ (Figure 1). The overall survival time in patients with luminal type $A$ showed significant differences with other subtypes (Figure 2), but the survival time after brain metastases in the various subtypes showed no significant differences (Table 1). These indicated that patients with HER-2 overexpression and TN had rapid disease progression, shor times of effective treatment, and short overall survival time, with poor prognosis particularly in patients with HER-2 overexpression.

\section{Prognostic factors in patients with brain metastases}

Table 1 Analysis of clinical characteristics and survival of 4 subtypes of breast cancer with brain metastasis in 80 patients ${ }^{a}$

\begin{tabular}{|c|c|c|c|c|c|c|c|c|}
\hline Group & Patient No. & $\begin{array}{c}\text { Age at initial } \\
\text { diagnosis (years) }\end{array}$ & $\begin{array}{l}\text { BM age } \\
\text { (years) }\end{array}$ & $\begin{array}{l}\text { Disease free } \\
\text { interval (months) }\end{array}$ & $\begin{array}{l}\text { PFS after } 1^{\text {st }} \text { line } \\
\text { treatment (months) }\end{array}$ & $\begin{array}{c}\text { Interval from initial } \\
\text { diagnosis to BM (months) }\end{array}$ & $\begin{array}{l}\text { Survival after } \\
\text { BM (months) }\end{array}$ & $\begin{array}{l}\text { Overall survival } \\
\text { (months) }\end{array}$ \\
\hline Overall & 80.0 & 44.5 & 48.5 & 20.0 & 9.0 & 30.0 & 8.0 & 41.5 \\
\hline Luminal $A$ & 30.0 & 41.0 & 46.0 & 25.5 & 11.0 & 46.5 & 8.0 & 63.0 \\
\hline Luminal B & 20.0 & 45.5 & 49.0 & 24.5 & 10.5 & 34.5 & 8.0 & 58.0 \\
\hline HER-2 & 16.0 & 50.0 & 52.0 & 9.0 & 8.0 & 20.5 & 7.0 & 25.0 \\
\hline TN & 14.0 & 44.5 & 48.0 & 11.5 & 8.0 & 27.0 & 8.0 & 33.0 \\
\hline$P$ & & 0.027 & 0.293 & 0.064 & 0.049 & 0.025 & 0.156 & 0.001 \\
\hline
\end{tabular}

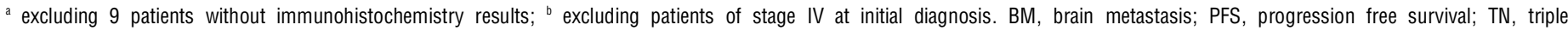
negative.

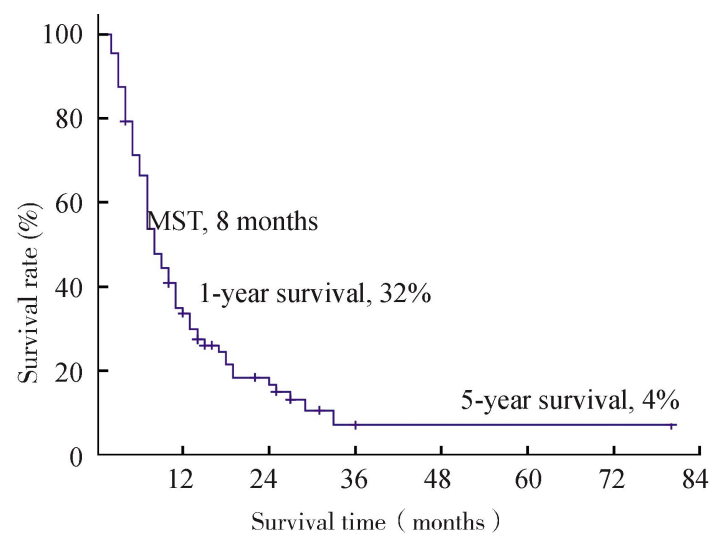

Figure 1 Overall survival of all patients

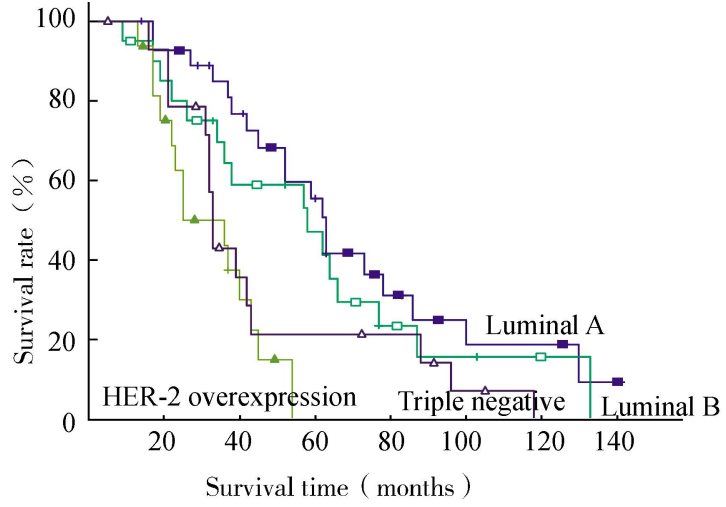

Figure 2 Overall survival of patients with different subtypes of breast cancer
Univariate analysis showed that performance status score, time to brain metastases, time from the first consultation to brain metastases, number of brain metastases, extracranial metastasis, treatment of brain metastases, endocrine therapy, and chemotherapy using taxane and capecitabine all had an impact on survival. The analysis of age and different subtypes indicated that patients younger than 55 years and patients with luminal type A disease had a tendency for good prognosis, but without statistical significance.

Multivariate analysis showed that performance status score, number of brain metastases, and the treatment of brain metastases with or without endocrine therapy had an impact on survival. Patients with performance status scores of 0 to 1 points, with single brain metastasis, receiving WBRT combined with 


\section{Chinese Journal of Cancer}

chemotherapy, and receiving endocrine therapy had a better prognosis (Table 2).

\section{Analysis of patients with luminal type disease with endocrine therapy}

Endocrine therapy had great significance in patients with positive hormone receptors. In this study the treatment and prognosis of patients with luminal types (including type $A$ and type B) were analyzed separately. In patients with luminal types of breast cancer with brain metastases, those with performance status scores of 0 to 1 points had a better prognosis, and radiotherapy and tamoxifen could improve survival. Patients with single metastasis and receiving chemotherapy had a tendency of good prognosis, and aromatase inhibitor therapy had no significant effect on survival (Table 3 ).

In this study 30 patients with previous adjuvant tamoxifen therapy were further analyzed. The median time of adjuvant tamoxifen therapy in 30 patients was 25 months (2-60 months), of which 25 patients also received sequential therapy with aromatase inhibitors. A total of 14 patients had recurrence or metastasis during adjuvant endocrine therapy and the median time to progression was 18 months (2-54 months). A total of 16 patients had recurrence or metastasis after the cessation of adjuvant endocrine therapy, and the median time from the cessation to recurrence or metastasis was 15 months (2-50 months). After the occurrence of brain metastases, the patients that received tamoxifen treatment had an obviously longer median survival time compared with those that did not receive tamoxifen (25 months vs. 6 months). Multivariate analysis showed that endocrine therapy was an independent prognostic factor (Figure 3). This indicate that in patients with luminal types

Table 2 Univariate and multivariate prognostic analysis of 87 patients $^{\mathrm{a}}$

\begin{tabular}{|c|c|c|c|c|c|}
\hline \multirow{2}{*}{ Prognostic factor } & \multirow{2}{*}{ Patient No. } & \multirow{2}{*}{ Median survival (months) } & \multirow{2}{*}{$95 \% \mathrm{Cl}$} & \multicolumn{2}{|c|}{$P$} \\
\hline & & & & Univariate & Multivariate \\
\hline$\overline{\text { Age (years) }}$ & & & & 0.159 & \\
\hline$>55$ & 39 & 7.0 & $5.90-8.09$ & & \\
\hline$\leqslant 55$ & 48 & 9.0 & $6.03-11.97$ & & \\
\hline Performance status score & & & & $<0.001$ & $<0.001$ \\
\hline $0-1$ & 67 & 10.0 & $7.5-12.32$ & & \\
\hline$>1$ & 22 & 3.0 & $0.28-4.79$ & & \\
\hline \multicolumn{6}{|l|}{ Subtype $^{b}$} \\
\hline Luminal A & 30 & 8.0 & $3.97-12.02$ & & \\
\hline Luminal B & 20 & 8.0 & $4.84-11.15$ & 0.156 & \\
\hline HER-2 & 16 & 7.0 & $4.40-9.59$ & & \\
\hline TN & 14 & 8.0 & $5.55-10.44$ & & \\
\hline Metastatic time & & & & 0.019 & 0.677 \\
\hline Initial or 1st line & 24 & 11.0 & $7.81-14.19$ & & \\
\hline 2nd line or later & 63 & 7.0 & $5.72-8.27$ & & \\
\hline Interval from initial diagnosis to $\mathrm{BM}$ & & & & 0.046 & 0.143 \\
\hline$>2$ years & 52 & 9.0 & $6.38-11.61$ & & \\
\hline$\leqslant 2$ years & 35 & 7.0 & $5.35-8.65$ & & \\
\hline \multicolumn{6}{|l|}{ Other sites of metastasis } \\
\hline Bone or visceral & 70 & 7.0 & $5.75-8.24$ & 0.026 & 0.355 \\
\hline None & 8 & 18.0 & $4.14-31.85$ & & \\
\hline Other site except bone or visceral & 9 & 13.0 & $0.00-30.81$ & & \\
\hline Number of BM sites & & & & 0.023 & 0.034 \\
\hline Isolated & 40 & 11.0 & $8.53-13.46$ & & \\
\hline Multiple & 47 & 7.0 & $6.18-7.81$ & & \\
\hline \multicolumn{6}{|l|}{ Treatment } \\
\hline Symptomatic & 21 & 5.0 & $2.75-7.24$ & $<0.001$ & $<0.001$ \\
\hline CT or WBRT alone & 29 & 7.0 & $5.54-8.45$ & & \\
\hline CT and WBRT & 39 & 11.0 & $8.47-13.55$ & & \\
\hline Capecitabine & & & & 0.032 & 0.448 \\
\hline Yes & 20 & 11.0 & $6.73-15.27$ & & \\
\hline No & 67 & 7.0 & $5.86-8.14$ & & \\
\hline Taxane & & & & 0.036 & 0.463 \\
\hline Yes & 20 & 15.0 & $7.35-22.65$ & & \\
\hline No & 67 & 7.0 & $5.86-8.14$ & & \\
\hline Endocrine therapy & & & & $<0.001$ & $<0.001$ \\
\hline Yes & 19 & 24.0 & $25.51-60.09$ & & \\
\hline No & 68 & 7.0 & $7.62-11.27$ & & \\
\hline
\end{tabular}

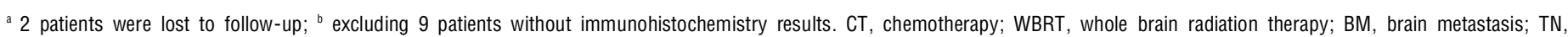
triple negative. 


\section{Chinese Journal of Cancer}

Table 3 Prognostic analysis of clinical characteristics and treatment in luminal subtypes of breast cancer with brain metastasis in 50 patients

\begin{tabular}{|c|c|c|c|c|c|}
\hline \multirow{2}{*}{ Prognostic factor } & \multirow{2}{*}{ Patient No. } & \multirow{2}{*}{ Median survival (months) } & \multirow{2}{*}{$95 \% \mathrm{Cl}$ (months) } & \multicolumn{2}{|c|}{$P$} \\
\hline & & & & Univariate & Multivariate \\
\hline Performance status score & & & & $<0.001$ & $<0.001$ \\
\hline $0-1$ & 38 & 11.0 & $3.59-18.41$ & & \\
\hline$>1$ & 12 & 3.0 & $1.88-4.11$ & & \\
\hline Number of BM sites & & & & 0.089 & \\
\hline Isolated & 20 & 11.0 & $8.82-13.18$ & & \\
\hline Multiple & 30 & 7.0 & $5.27-8.73$ & & \\
\hline WBRT & & & & $<0.001$ & 0.004 \\
\hline Yes & 37 & 11.0 & $6.67-15.33$ & & \\
\hline No & 13 & 4.0 & $2.91-5.08$ & & \\
\hline Chemotherapy & & & & 0.167 & \\
\hline Yes & 21 & 11.0 & $9.51-12.48$ & & \\
\hline No & 29 & 7.0 & $4.48-9.52$ & & \\
\hline Tam & & & & $<0.001$ & 0.002 \\
\hline Yes & 14 & 24.0 & none & & \\
\hline No & 36 & 7.0 & $5.39-8.60$ & & \\
\hline $\mathrm{Al}$ & & & & 0.738 & \\
\hline Yes & 6 & 7.0 & $0-18.55$ & & \\
\hline No & 44 & 8.0 & $4.81-11.19$ & & \\
\hline
\end{tabular}

WBRT, whole brain radiation therapy; BM, brain metastasis; Tam, tamoxifen; Al, aromatase inhibitor.

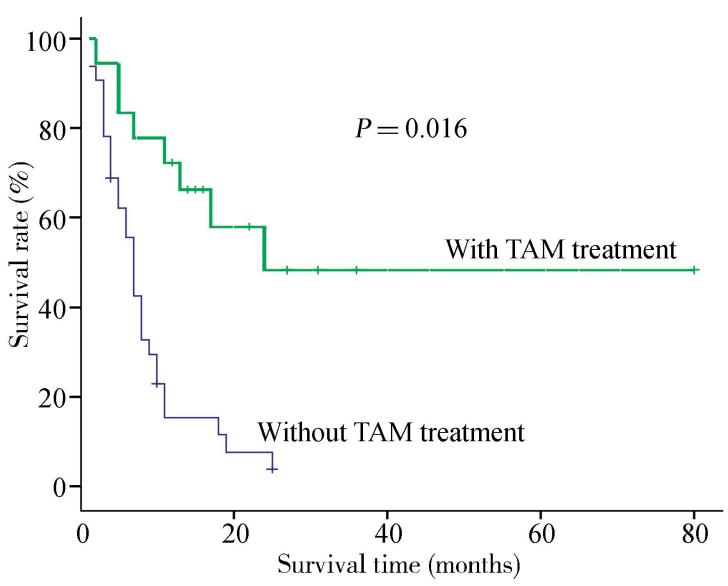

Figure 3 Tamoxifen treatment in patients with brain metastasis of luminal subtypes with previously used adjuvant tamoxifen

of disease that received previous adjuvant tamoxifen therapy, the continued use of tamoxifen still had a survival benefit (Table 4).

\section{Discussion}

In 2000, breast cancer was divided into 5 subtypes by Perou et al. ${ }^{[12]}$ through cDNA microarray technology: luminal $A$, luminal $B$, HER-2 overexpressing, basal-like, and normal breast like, and different subtypes had significant differences in prognosis. Because genotyping needs fresh specimens and high technology, it cannot be used in clinic routinely, and immunohistochemistry is often clinically used for typing. A large body of research suggests that HER-2 gene overexpression increases proliferation, survival, anti-apoptosis, migration, and invasive capabilities of tumor cells and is an important risk factor for brain metastases from breast cancer ${ }^{[8-11]}$. TN breast cancer is characterized by a high rate of early recurrence, rapid progression, a high rate of visceral metastasis, and easy metastasis of the central nervous system ${ }^{[5-7]}$. The results of this study also showed that patients with HER-2 overexpression and TN breast cancer had poor prognosis, early brain metastases, and rapid disease progression after treatment.

In this study, performance status scores, number of metastases, and treatment were independent prognostic factors for patients with breast-cancer related brain metastasis. Performance status score is a prognostic factor for many tumors reflecting the overall survival of patients, and is an important reference for assessing patients and clinical decision-making. Multiple metastases indicate a high proliferation rate and strong invasions of tumor cells, or a weak antitumor immunity of the body. Compared with multiple metastases, a single metastasis has more of a chance to be cured through further surgery or stereotactic radiotherapy.

This study showed that age and accompanied metastases had no significant effect on the prognosis. In general, age is an important prognostic factor in patients with breast cancer. In newly diagnosed patients, being younger than 35 years is a high risk factor for breast cancer recurrence. But in patients with brain metastases from breast cancer, the young do not necessarily represent short survival time. Some studies suggested that age was not an independent prognostic factor in patients with breast cancer metastasis ${ }^{[5,6]}$. This study also found no survival differences among patients of different ages, but patients younger than 55 years had a better trend. The reason may be that older patients have poorer physical condition and immune function, often with hypertension, diabetes, and other chronic diseases, so the 


\section{Chinese Journal of Cancer}

Table 4 Prognostic analysis of clinical character and treatment in patients with brain metastasis of luminal subtype with previously used adjuvant tamoxifen

\begin{tabular}{|c|c|c|c|c|c|}
\hline \multirow{2}{*}{ Prognostic factor } & \multirow{2}{*}{ Patient No. } & \multirow{2}{*}{ Median survival (months) } & \multirow{2}{*}{$95 \% \mathrm{Cl}$ (months) } & \multicolumn{2}{|c|}{$P$} \\
\hline & & & & Univariate & Multivariate \\
\hline Performance status score & & & & $<0.001$ & 0.004 \\
\hline $0-1$ & 23 & 13.0 & $0.0-31.90$ & & \\
\hline$>1$ & 7 & 1.0 & $0.43-5.56$ & & \\
\hline Number of BM site & & & & 0.011 & 0.062 \\
\hline isolated & 12 & 25.0 & none & & \\
\hline multiple & 18 & 6.0 & $3.23-8.77$ & & \\
\hline WBRT & & & & $<0.001$ & 0.075 \\
\hline Yes & 20 & 13.0 & $0.0-38.32$ & & \\
\hline No & 10 & 4.0 & $3.05-4.94$ & & \\
\hline Chemotherapy & & & & 0.415 & \\
\hline Yes & 12 & 10.0 & $4.91-15.09$ & & \\
\hline No & 18 & 6.0 & $3.93-8.06$ & & \\
\hline Tam & & & & 0.001 & 0.016 \\
\hline Yes & 22 & 25.0 & none & & \\
\hline No & 8 & 6.0 & $3.71-8.29$ & & \\
\hline
\end{tabular}

WBRT, whole brain radiation therapy; BM, brain metastasis; Tam, tamoxifen.

performance status is relatively poor, which to some extent affects prognosis. In patients accompanied with extracranial metastasis, some research reported that patients with visceral metastasis had poor prognosis ${ }^{[2,13]}$. However, in this study, the corresponding multivariate analysis showed no statistical significance.

The main treatments for brain metastases from breast cancer include WBRT, chemotherapy, endocrine therapy, targeted therapy, surgery, SRS, and so on. This study showed that on the whole, WBRT combined with systemic chemotherapy had the best results $(P<0.001)$. In the past 50 years, WRBT has been considered the standard treatment for brain metastases. It can delay or prevent the progress of nerve damage, relieve symptoms, and improve patient quality-of-life. The local control rate with WBRT can be up to $90 \%-100 \%$. The role of chemotherapy is still controversial and its limitations currently include the following. First, only a few drugs can enter the brain because of the blood-brain barrier. Secondly, $\mathrm{P}$-glycoprotein is highly expressed in brain capillary endothelial cells, which can transport chemotherapy drugs with various structures and functions out of the cells ${ }^{[14]}$. Finally, drugs that can get through the blood-brain barrier are often not sensitive to the tumors, or have been used in previous treatment, so the tumors have become resistant. Currently, the benefit of chemotherapy combined with radiotherapy is considered because the blood-brain barrier is destroyed after radiotherapy so drugs can get into the brain more easily, and systemic chemotherapy is an effective means to control other lesions. In chemotherapy drugs, capecitabine has been a research hotspot in recent years ${ }^{[15-18]}$, but in this study it was not found to improve survival.

Because of the small number of patients, targeted therapy, SRS, and surgery were not analyzed in this study, and these methods also have some effect in specific patients. It is reported that the small-molecule tyrosine kinase inhibitor lapatinib has good therapeutic effect in patients with HER-2 positive breast cancer with brain metastases ${ }^{[19,20]}$. The median survival time of patients with breast-cancer related brain metastases treated by SRS can be up to $10.5-13$ months $^{[21,22]}$, and in the present study 1 patient received $\gamma$-knife treatment and achieved the longest survival time in the whole group (80 months).

Endocrine therapy is currently considered to have a slow onset and is not the first choice in patients with brain metastases from breast cancer. In commonly used drugs, tamoxifen can pass through the blood-brain barrier and has a certain effect in patients with brain metastasis from breast cancer ${ }^{[23]}$. Aromatase inhibitors play an antitumor effect through further reducing the estrogen level in postmenopausal patients, but have no direct effect on tumor cells. Some research has reported that letrozole displayed a certain degree of activity in treatment ${ }^{[24]}$. The new anti-estrogen drug fulvestrant was found not to pass through blood-brain barrier in earlier studies. It is interesting that the results of this study showed that tamoxifen could improve the prognosis of patients with brain metastases from luminal-type breast cancer, regardless of whether adjuvant therapy with tamoxifen was used. However, these conclusions have certain limitations because of the small number of patients and the retrospective analysis. In clinical work, after the diagnosis of brain metastases, patients first receive WBRT and systemic chemotherapy, followed by endocrine therapy. After 2-5 months of chemotherapy and radiotherapy, treatment-sensitive patients have a reduction or disappearance of tumor lesions and improved performance status and can receive further treatment, while the patients not sensitive to treatment often deteriorate rapidly. Therefore, the patients accepting tamoxifen usually have good performance status and are sensitive to previous treatment. Those who do not receive tamoxifen usually have disease that cannot be controlled by radiotherapy or chemotherapy and die before tamoxifen therapy, which may be a bias factor. In addition, in this study, nearly half of the patients (14) had progression with endocrine therapy. Clinically, disease 


\section{Chinese Journal of Cancer}

progression in patients after palliative treatment is not uncommon. Can patients still benefit from tamoxifen after progression? On the other hand, taking into account the priority of endocrine therapy, can patients with negative hormone receptor disease (including TN and HER-2) also benefit from tamoxifen therapy? Prospective study is needed to observe the efficacy of tamoxifen.

At present it has been confirmed that preventive WBRT can improve the overall survival rate of patients with small-cell lung cancer after complete remission by chemotherapy ${ }^{[25]}$. In clinic, preventive intrathecal injection has become a common treatment in patients at high risk for lymphoma and leukemia. For those patients with breast cancer with a high risk of brain metastases, learning from the treatment experience of small-cell lung cancer and lymphoma, whether preventive radiotherapy and intrathecal injection can be used to prevent or delay the occurrence of brain metastases and improve overall survival remains to be further studied.

In summary, patients with brain metastases from breast cancer have short survival times, and patients with the HER-2 and TN types have particularly poor prognosis. Specific multidisciplinary treatments should be taken depending on the specific circumstances of patients with breast-cancer related brain metastases. WBRT combined with chemotherapy is the appropriate treatment, and in patients with positive hormone receptors, tamoxifen therapy can prolong survival. Targeted therapy, SRS, and surgery also have a certain role for specific patients. Prospective randomized controlled studies can help to further understand the risk factors, prognostic factors, and treatments for patients with breast cancer brain metastasis. In patients at high risk for brain metastases, whether routine brain screening should be used regularly and whether preventive treatment should be used to prevent or delay the occurrence of brain metastases and improve overall survival still needs further study.

\section{References}

[1] Barnholtz-Sloan JS, Sloan AE, Davis FG, et al. Incidence proportions of brain metastases in patients diagnosed (1973 to 2001) in the Metropolitan Detroit Cancer Surveillance System [J]. J Clin Oncol, 2004,22(14):2865-2872

[2] Lin NU, Bellon JR, Winer EP. CNS metastases in breast cancer [J]. J Clin Oncol, 2004,22(17):3608-3617

[3] Patanaphan V, Salazar OM, Risco R. Breast cancer: Metastatic patterns and their prognosis [J]. South Med J, 1988,81(9):1109-1112.

[4] Tsukada Y, Fouad A, Pickren JW, et al. Central nervous system metastasis from breast carcinoma. Autopsy study [J]. Cancer, 1983,52 (12):2349-2354.

[5] Lin NU, Claus E, Sohl J, et al. Sites of Distant recurrence and clinical outcomes in patients with metastatic triple-negative beast cancer [J]. Cancer, 2008,113(10):2638-2645

[6] Dawood S, Broglio K, Esteva FJ, et al. Survival among women with triple receptor-negative breast cancer and brain metastases [J]. Ann Oncol,
2009,20(4):621-627

[7] Nam BH, Kim SY, Han HS, et al. Breast cancer subtypes and survival in patients with brain metastases [J]. Breast Cancer Res, 2008,10(1):R20.

[8] Gabos Z, Sinha R, Hanson J, et al. Prognostic significance of human epidermal growth factor receptor positivity for the development of brain metastasis after newly diagnosed breast cancer [J]. J Clin Oncol, 2006, 24(36):5658-5663

[9] Pestalozzi BC, Zahrieh D, Price $\mathrm{KN}$, et al. Identifying breast cancer patients at risk for central nervous system (CNS) metastases in trials of the International Breast Cancer Study Group (IBCSG) [J]. Ann Oncol, 2006, 17(6):935-944

[10] Hicks DG, Short SM, Prescott NL, et al. Breast cancers with brain metastases are more likely to be estrogen receptor negative, express the basal cytokeratin CK5/6, and over express HER2 or EGFR [J]. Am J Surg Pathol, 2006,30(9):1097-1104.

[11] Tham, YL, Sexton K, Kramer R, et al. Primary breast cancer phenotypes associated with propensity for central nervous system metastases [J] Cancer, 2006, 107(10):2521-2522

[12] Perou CM, Sфrlie T, Eisen MB, et al. Molecular portraits of human breast tumors [J]. Nature, 2000,406(6797):747-752.

[13] Miller KD, Weathers T, Haney LG, et al. Occult central nervous system involvement in patients with metastatic breast cancer: prevalence, predictive factors and impact on overall survival [J]. Ann Oncol, 2003,14 (7):1072-1077.

[14] Fenner MH, Possinger K. Chemotherapy for breast cancer brain metastases [J]. Onkologie, 2002,25(5):474-479.

[15] Ekenel M, Hormigo AM, Peak S, et al. Capecitabine therapy of central nervous system metastases from breast cancer [J]. Neurooncol, 2007,85 (2):223-227.

[16] Chargari C, Kirova YM, Diéras V, et al. Concurrent capecitabine and whole-brain radiotherapy for treatment of brain metastases in breast cancer patients [J]. J Neurooncol, 2009,93(3):379-384

[17] Fabi A, Vidiri A, Ferretti G, et al. Dramatic regression of multiple brain metastases from breast cancer with Capecitabine. another arrow at the bow [J]. Cancer Invest, 2006,24(4):466-468.

[18] Wang ML, Yung WK, Royce ME, et al. Capecitabine for 5-fluorouracilresistant brain metastases from breast cancer [J]. Am J Clin Oncol, 2001,24(4):421-424.

[19] Lin NU, Carey LA, Liu MC, et al. Phase II trial of lapatinib for brain metastases in patients with human epidermal growth factor receptor 2 positive breast cancer [J]. J Clin Oncol, 2008,26(12):1993-1999.

[20] Lin NU, Diéras V, Paul D, et al. Multicenter phase ॥ study of lapatinib in patients with brain metastases from HER2-positive breast cancer [J]. Clin Cancer Res, 2009,15(4):1452-1459

[21] Chidel MA, Suh JH, Reddy CA, et a1.Application of the recursive partitioning analysis and evaluation of the use of whole brain radiation among patients treated with stereotaetic radiosurgery for newly diagnosed brain metastases [J]. Int J Radiat Oncol Biol Phys, 2000,47(4):993-999.

[22] Firlik KS, Kondziolka D, Flickinger JC, et al.Stereotactic radiosurgery for brain metastases from breast cancer [J]. Ann Surg Oncol, 2000,7(5): 333-338.

[23] Carey RW, Davis JM, Zervas NT. Tamoxifen-induced regression of cerebral metastases in breast carcinoma [J]. Cancer Treat Rep, 1981,65 $(9-10): 793-795$

[24] Madhup R, Kirti S, Bhatt ML, et al. Letrozole for brain and scalp metastases from breast cancer - a case report. [J]. Breast, 2006,15(3): $440-442$

[25] Slotman B, Faivre-Finn C, Kramer G, et al. Prophylactic cranial irradiation in extensive small-cell lung cancer [J]. N Engl J Med, 2007,357(7):664672 . 